\title{
Development of Protocol for Korean Lung Cancer Screening Project (K-LUCAS) to Evaluate Effectiveness and Feasibility to Implement National Cancer Screening Program
}

\author{
Jaeho Lee, $\mathrm{PhD}^{1}$ \\ Juntae Lim, MD', \\ Yeol Kim, MD, PhD1,2 \\ Hyae Young Kim, MD, $\mathrm{PhD}{ }^{3}$ \\ Jin Mo Goo, MD, PhD 4 \\ Choon-Taek Lee, MD, PhD 5 \\ Seung Hun Jang, MD, $\mathrm{PhD}{ }^{6}$ \\ Won-Chul Lee, MD, $\mathrm{PhD}^{7}$ \\ Chan Wha Lee, MD, $\mathrm{PhD}^{3}$ \\ Jin Young An, MD, PhD \\ Ki Dong Ko, MD, MPH ${ }^{9}$ \\ Min Ki Lee, MD, PhD 10 \\ Kui Son Choi, PhD',2 \\ Boyoung Park, MD, PhD ${ }^{1,2}$ \\ Duk Hyoung Lee, MD, PhD ${ }^{1}$
}

*A list of author's affiliations appears at the end of the paper.

\begin{abstract}
Purpose
To reduce lung cancer mortality, lung cancer screening was recommended using low-dose computed tomography (LDCT) to high-risk population. A protocol for multicenter lung cancer screening pilot project was developed to evaluate the effectiveness and feasibility of lung cancer screening to implement National Cancer Screening Program in Korea.
\end{abstract}

\begin{abstract}
Materials and Methods
Multidisciplinary expert committee was comprised to develop a standardized protocol for Korean Lung Cancer Screening Project (K-LUCAS). K-LUCAS is a population-based single arm trial that targets high-risk population aged 55-74 years with at least 30 pack-year smoking history. LDCT results are reported by Lung-RADS suggested by American Radiology Society. Network-based system using computer-aided detection program is prepared to assist reducing diagnostic errors. Smoking cessation counselling is provided to all currently smoking participants. A small pilot test was conducted to check the feasibility and compliance of the protocols for K-LUCAS.
\end{abstract}

\section{Results}

In pilot test, 256 were participated. The average age of participants was 63.2 years and only three participants (1.2\%) were female. The participants had a smoking history of 40.5 pack-year on average and 53.9\% were current smokers. Among them, $86.3 \%$ had willing to participate in lung cancer screening again. The average willingness to quit smoking among current smokers was $12.7 \%$ higher than before screening. In Lung-RADS reports, 10 (3.9\%) were grade 3 and nine (3.5\%) were grade 4 . One participant was diagnosed as lung cancer.

\section{Conclusion}

The protocol developed by this study is assessed to be feasible to perform K-LUCAS in multicenter nationwide scale.

\section{Introduction}

In a last few decades, lung cancer has been the most frequent cancer in the world. Lung cancer contributes to $12.9 \%$ of the total cancers in the world and responsible for approximately one fifth of the total cancer death worldwide. Over 1.8 million people were newly diagnosed with lung cancers in 2012 and about $58.4 \%$ of these cancers were occurred in the less developed regions [1]. According to Korean National
Key words

Lung neoplasms, Mass screening,

Low-dose computed tomography, Smokers, Pilot test 


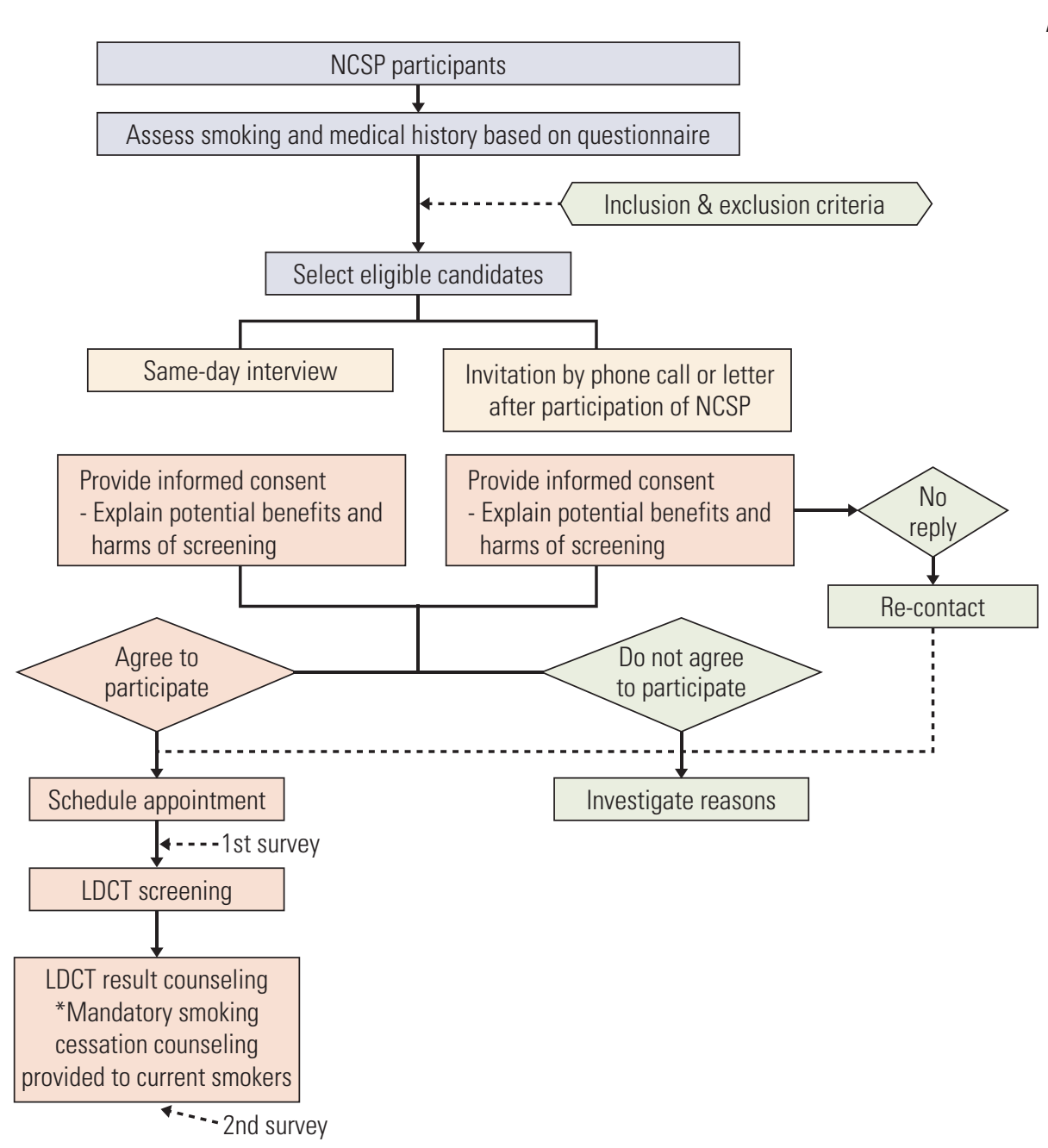

Fig. 1. A summary of lung cancer screening procedure in Korean Lung Cancer Screening Project (K-LUCAS). (A) A summary of lung cancer screening procedure for National Cancer Screening Program (NCSP) participants. (Continued to the next page)

detected lung cancers, $26(96 \%)$ were resectable whereas only 30 of the 59 tumors $(51 \%)$ detected by chest radiography were resectable [6,7].

Despite some encouraging results in the early studies, however, the effectiveness of LDCT screening, especially on the mortality reduction, has been remained doubtful until recently. In 2011, The National Lung Screening Trial (NLST) in the United States has produced a supportive landmark result that screening with LDCT targeted to high-risk smoking groups (age, 55 to 74 years; current smokers or former smokers within the past 15 years with at least 30 pack-year smoking history) reduced lung cancer mortality by $20 \%$ [8]. Building on the results of NLST, in December 2013, the U.S. Preventive Services Track Force (USPSTF) announced lung cancer screening recommendation for high-risk individuals [9] and in February 2015, The Centers for Medicare \& Medi- caid Services (CMS) issued a medicare coverage of lung cancer screening [10].

Based on the evidence in the United States, Korean National Cancer Center (NCC) has developed and published the guideline of lung cancer screening using LDCT for high-risk populations in 2015 [11]. The guideline recommends annual LDCT screening for high-risk smoking groups aged 55 to 74 years, with at least 30 pack-year smoking history and current smokers or former smokers who quit smoking within 15 years.

In 2016, Korean Ministry of Health and Welfare announced to launch The Korean Lung Cancer Screening project (K-LUCAS) in accordance with NCC's guideline of lung cancer screening using LDCT. K-LUCAS is a nationwide, multicenter, prospective study to evaluate the effectiveness and feasibility of lung cancer screening with LDCT for considering 


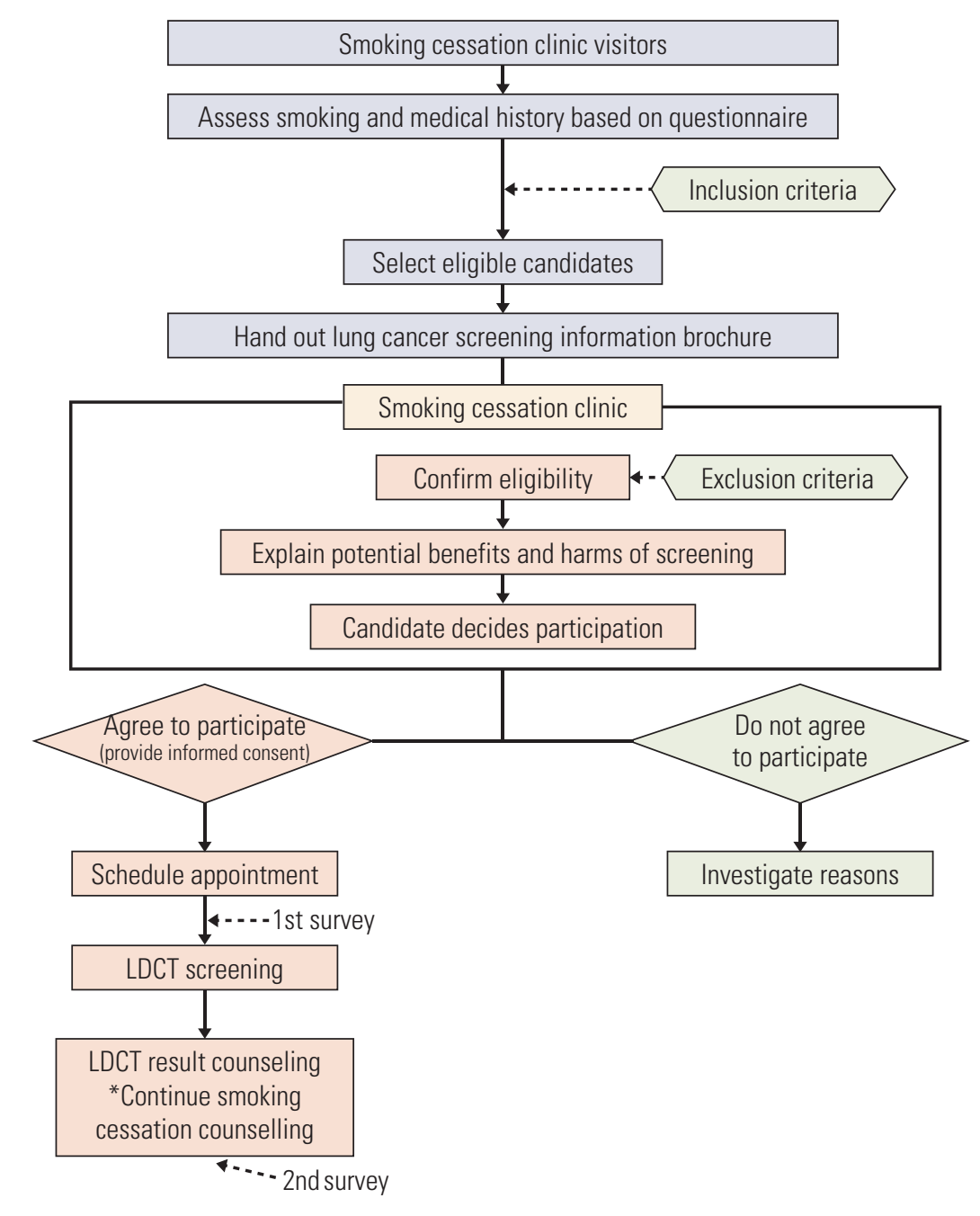

\section{B}

Fig. 1. (Continued from the previous page) (B) A summary of lung cancer screening procedure for smoking cessation clinic visitors. LDCT, low-dose computed tomography.

implementation of a national lung cancer screening program in Korea.

Although previous studies have shown that lung cancer screening with LDCT could help early lung cancer detection and hence could reduce cancer mortality, it is yet to be clear about how to implement an effective and efficient population-based screening program [12]. K-LUCAS evaluates the feasibility to select high-risk target group for screening precisely from the general population and the capability to reduce false-positive findings in screening results by the standardized guideline with quality control through network based computer assistant program. K-LUCAS is the first Asian population-based nationwide trial in testing the effectiveness and efficiency of lung cancer screening using LDCT.

The objective of this article is to provide a detailed descrip- tion of protocol developed for population-based lung cancer screening project in Korea, and to report the results of a small pilot test which conducted in four regional cancer hospitals to check the feasibility and compliance of the protocol for K-LUCAS.

\section{Materials and Methods}

\section{Development of K-LUCAS protocol}

Multidisciplinary expert committee was comprised to develop a standardized protocol for K-LUCAS. The commit- 
tee is consist of multidisciplinary experts in various fields of lung cancer screening including pulmonology, radiology, family medicine, preventive medicine and statistics to enhance an integration of expertise in designing protocols and procedures.

The participants are selected in accordance with NCC's guideline of lung cancer screening using LDCT for high-risk populations. The inclusion criteria for K-LUCAS are decided as follows: (1) 55-74 years aged current smokers or former smokers (who quit smoking within 15 years) and (2) at least 30 pack-years smoking history.

The exclusion criteria are as follows: (1) past history of lung cancer; (2) unable to move without help (Eastern Cooperative Oncology Group score 2 or higher); (3) on treatment for tuberculosis, pneumonia and interstitial lung disease; (4) diagnosis of any cancers within 5 years (exception: thyroid cancer and skin cancer); and (5) chest CT performed within 6 months.

Each criterion is carefully evaluated by our team of multidisciplinary experts. For example, the exclusion criteria exclude individuals who are diagnosed as any cancer within the last 5 years, because chest CT would be examined for the cancer patients regularly after first diagnosis with exception of thyroid cancer and skin cancer. Such exclusion criterion, therefore, prevents any unnecessary repetition of CT screening.
The participants in K-LUCAS are recruited from the visitors to participate in National Cancer Screening Program or smoking cessation services. Fig. 1 provides a summary of lung cancer screening procedure from the selection of participants to the result counselling through these two paths. The participants of K-LUCAS are selected based on the questionnaire that is completed in prior to participating in National Cancer Screening Program or smoking cessation services in accordance with the inclusion and exclusion criteria. The questionnaire consists of a series of questions for the purpose of gathering information on demographics, smoking status, smoking history and health status including cancer history. If the candidate meets the selection criteria, investigators carefully explain the benefits and harms of the lung cancer screening using LDCT. If the candidate agrees on lung cancer screening participation, an informed consent form is obtained and LDCT screening date is scheduled and confirmed. Surveys for participants are conducted on the day of screening and on the other day when the participants visited the hospital for counselling LDCT results. Participant satisfaction on general lung cancer screening processes, psychological harms of lung cancer before and after screening and an impact of lung cancer screening on smoking cessation motivation are investigated from these surveys.

K-LUCAS also provide a smoking cessation counselling to current smokers. This is done on the day of participant's visit

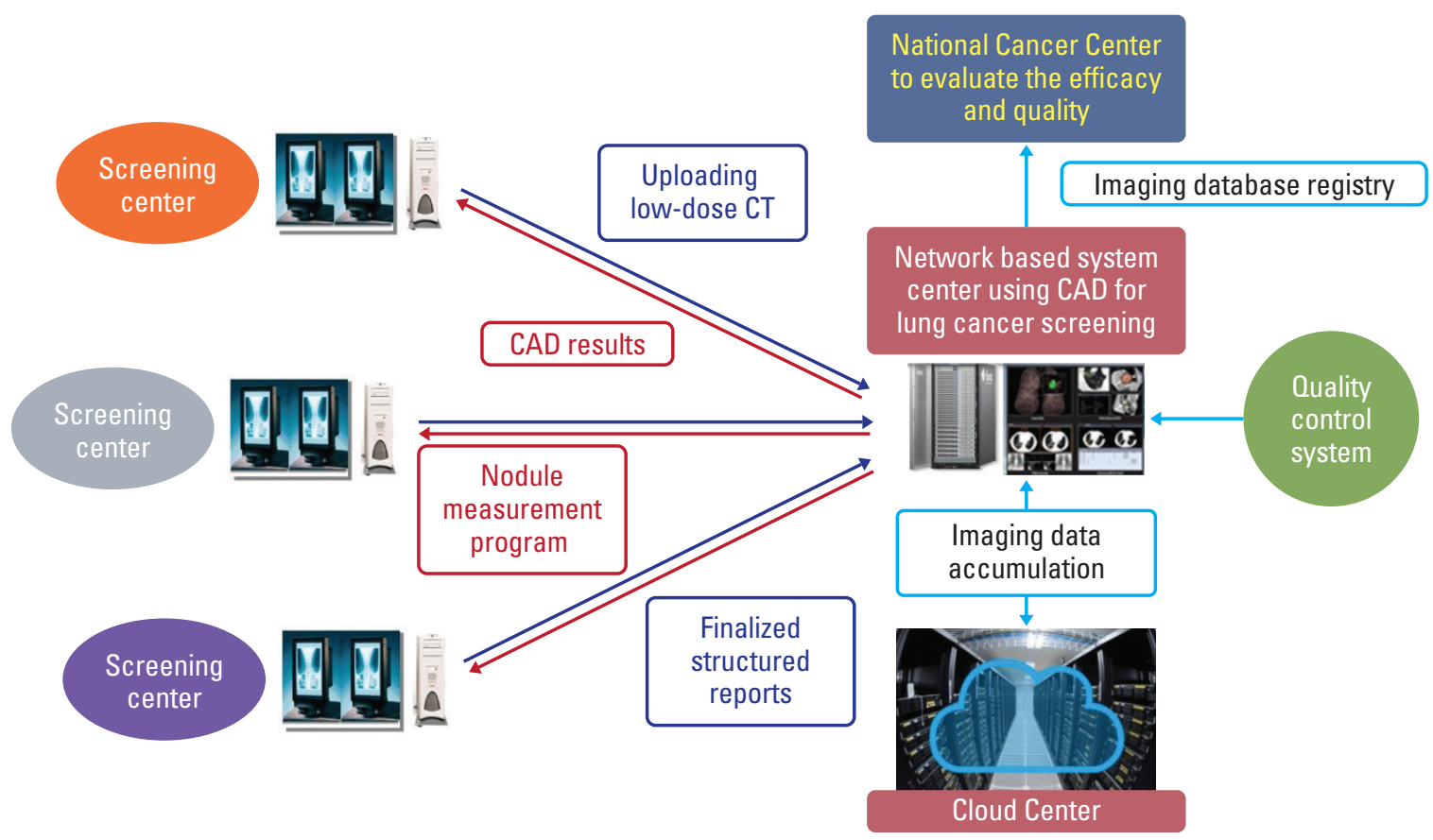

Fig. 2. Network-based diagnosis supporting system using computer-aided detection (CAD) program in Korean Lung Cancer Screening Project (K-LUCAS). CT, computed tomography. 


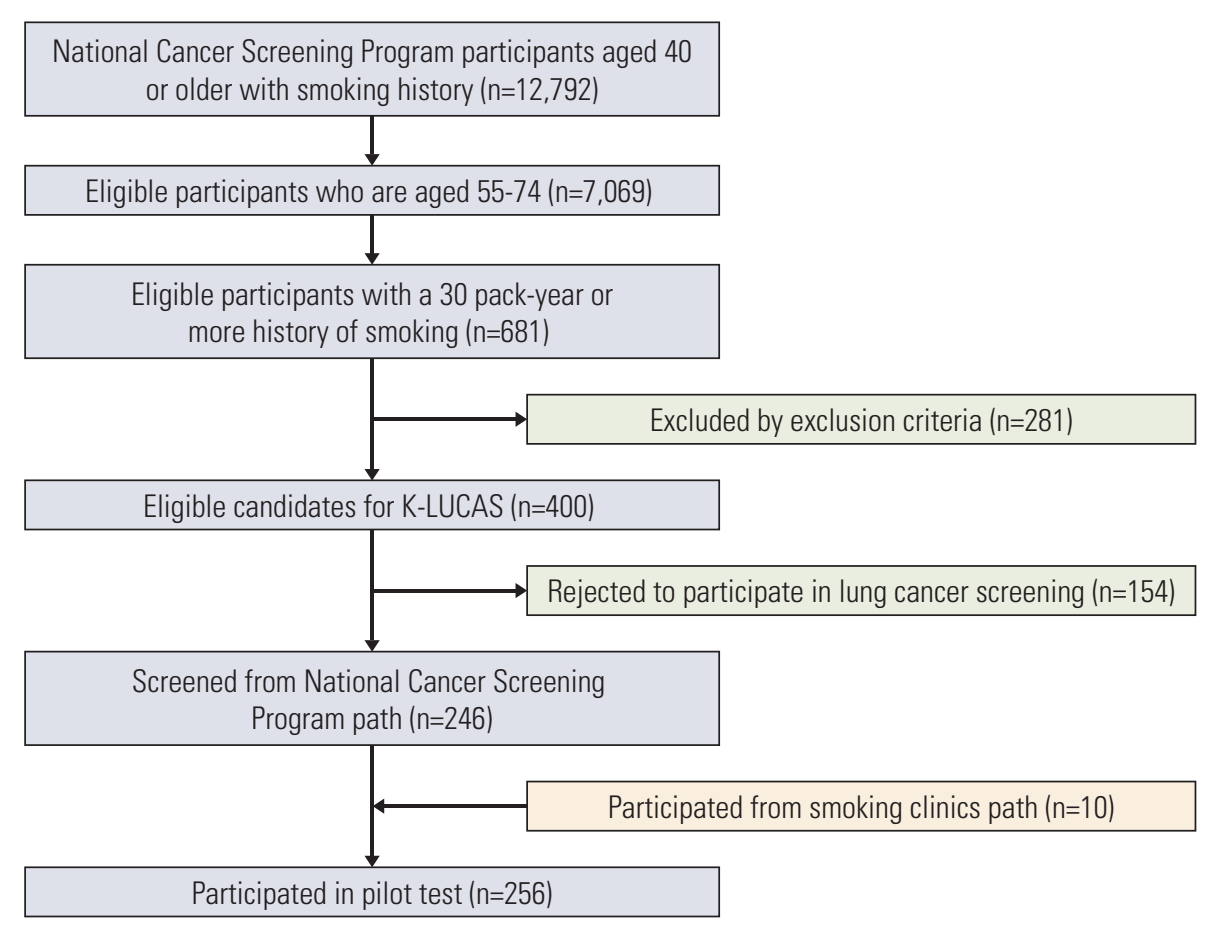

Fig. 3. Participant flow to screening in pilot test. K-LUCAS, Korean Lung Cancer Screening Project.

for receiving LDCT screening result counselling.

The LDCT screening results are evaluated by radiologists in accordance with lung imaging reporting and data system (Lung-RADS) suggested by American Radiology Society. Lung-RADS category 1 and 2 are classified as negative results and Lung-RADS category 3 and 4 are classified as positive results [13]. False-positives are defined as non-cancer diagnosis from total positive findings. Lung-RADS enables a standard follow-up screening procedure and treatment system [14,15]. Multidisciplinary expert committee has also agreed to use network-based diagnosis supporting system using computer-aided detection $(\mathrm{CAD})$ program to reduce missing lung nodules in LDCT screening. Previous studies have shown that $\mathrm{CAD}$ system has a potential to provide additional information to radiologists and hence would likely reduce diagnostic errors and increase lung nodule detection sensitivity [16]. Fig. 2 provides a summary of network-based diagnosis supporting system with CAD.

\section{Pilot test}

A small pilot test was conducted in four regional cancer hospitals to evaluate the feasibility and compliance of the protocol developed for K-LUCAS from October 2016 to March 2017. Participants in pilot test are selected from visitors participated in National Cancer Screening Program or smoking cessation service in four regional cancer hospitals.

We primarily considered following three features to evaluate the feasibility of K-LUCAS protocols. First, we investigated the proportion of eligible candidates that are agreed and rejected to participate in lung cancer screening. Second, we evaluated whether the positive rates are comparable with the rate of previously reported lung cancer screening trials. A comparable positive rates would be a good indicator of the capability of distinguishing pulmonary nodules from tuberculosis granulomas or scares in tuberculosis prevalent population in Korea [17].

Third, psychological harm from lung cancer screening is assessed. A new approach to reduce psychological harm would be necessary if participating in lung cancer screening caused too much psychological harm. We have used participant's anxiety of having lung cancer as a proxy to evaluate psychological harm of screening [18]. Anxiety of having lung cancer is a subjective measure on the score $0-10,0$ being no anxiety of having lung cancer and 10 indicates the highest degree of anxiety for lung cancer.

In addition to above three feasibility features, we also evaluated the effectiveness of smoking cessation counselling which provided to all current smokers participated in lung cancer screening. Negative finding from lung cancer screening can give reassurance for smoking. K-LUCAS thus analysed the effectiveness of smoking counselling by comparing 
Table 1. General characteristics of participants in pilot test by smoking status

\begin{tabular}{|c|c|c|c|}
\hline Characteristic & Current smokers ( $\mathrm{n}=138$ ) & Former smokers $(\mathrm{n}=118)$ & $p$-value ${ }^{a)}$ \\
\hline \multicolumn{4}{|l|}{ Sex } \\
\hline Male & $136(98.6)$ & $117(99.2)$ & 0.559 \\
\hline Female & $2(1.4)$ & $1(0.8)$ & \\
\hline \multicolumn{4}{|l|}{ Age group (yr) } \\
\hline $55-64$ & $106(76.8)$ & $80(67.8)$ & 0.107 \\
\hline $65-74$ & $32(23.2)$ & $38(32.2)$ & \\
\hline \multicolumn{4}{|c|}{ Smoking history (pack year) } \\
\hline$<40$ & $59(42.8)$ & $74(62.7)$ & 0.001 \\
\hline$\geq 40$ & $79(57.2)$ & $44(37.3)$ & \\
\hline \multicolumn{4}{|c|}{ Years of education } \\
\hline$\leq 9$ & $40(29.0)$ & $28(23.7)$ & 0.609 \\
\hline $10-12$ & $46(33.3)$ & $41(34.7)$ & \\
\hline$\geq 13$ & $47(34.1)$ & $45(38.1)$ & \\
\hline No answer & $5(3.6)$ & $4(3.5)$ & \\
\hline \multicolumn{4}{|c|}{ Monthly household income (million won) } \\
\hline$\leq 199$ & $53(38.4)$ & $36(30.5)$ & 0.116 \\
\hline 200-399 & $41(29.7)$ & 49 (41.5) & \\
\hline$\geq 400$ & $36(26.1)$ & $25(21.2)$ & \\
\hline No answers & $8(5.8)$ & $8(6.8)$ & \\
\hline
\end{tabular}

Values are presented as number (\%). a) Analyzed by chi-square test.

participant's willingness to quit smoking before and after lung cancer screening. Willingness to quit smoking is a subjective measure on the score 0-10 where score zero indicates no intention to quit smoking and score 10 indicates the highest degree of willingness to quit smoking.

Participants' satisfaction on screening processes and willingness to participate again in lung cancer screening in future were also surveyed to assess the participant compliance with the developed protocol for K-LUCAS.

\section{Statistical analysis}

The association between demographic characteristics and smoking status were evaluated using chi-square test. The changes in willingness to quit smoking and the changes in participant's anxiety of having lung cancer before and after LDCT screening were evaluated by paired $t$ test. All statistical analyses were performed using STATA software ver. 14 (Stata Corp. L.P., College Station, TX).

\section{Ethical statement}

Informed consent was obtained from all study participants in pilot test, and this study was approved by the Institutional Review Board of the National Cancer Center, Korea (IRB No. NCC2016-0255).

\section{Results}

\section{Participants in pilot test}

Fig. 3 shows participant flow to participation in the pilot test. A total of 12,792 visitors aged 40 or older with smoking history participated in national cancer screening and 7,069 (55.3\%) of the total visitors met our age criteria. Among these 7,069 visitors, $681(9.6 \%)$ had over 30 pack-year smoking history. After $281(41.3 \%)$ were excluded by exclusion criteria, 400 national screening program visitors were eligible to lung cancer screening. After the initial contact to eligible visitors, $154(21.6 \%)$ rejected to participate and the remaining 246 visitors were participated in pilot test. The participant rates to screening after initial contact to eligible 400 national screening program visitors was $61.5 \%$. Additionally, 10 visitors of smoking cessation clinics were also participated in pilot test.

Table 1 summarizes the socio-demographic characteristics of total 256 participants in the pilot test by their current smoking status. Only three participants were female and of the 256 participants, 138 (53.9\%) were current smokers and $118(46.1 \%)$ were former smokers. Seventy-nine participants $(57.2 \%)$ who currently smoke had a smoking history of more than 40 pack-years but only 44 former smokers (37.3\%) had a smoking history over 40 pack-years. There was more number of older participants in former smokers group than cur- 
Table 2. Lung cancer screening results reported based on Lung-RADS by screening hospitals in pilot test

\begin{tabular}{|c|c|c|c|c|c|}
\hline Lung-RADS & $\begin{array}{c}\text { BS } \\
(n=42)\end{array}$ & $\begin{array}{c}\text { CB } \\
(n=22)\end{array}$ & $\begin{array}{c}\mathrm{IC} \\
(\mathrm{n}=100)\end{array}$ & $\begin{array}{c}\text { NCC } \\
(n=92)\end{array}$ & $\begin{array}{c}\text { Total } \\
(n=256)\end{array}$ \\
\hline Negative & & & & & $237(92.6)$ \\
\hline Cat 1 & $21(50.0)$ & $7(31.8)$ & $35(35.0)$ & $83(90.2)$ & $146(57.0)$ \\
\hline Cat 2 & $17(40.5)$ & $15(68.2)$ & $53(53.0)$ & $6(6.5)$ & $91(35.5)$ \\
\hline Positive & & & & & $19(7.4)$ \\
\hline Cat 3 & $1(2.4)$ & - & $9(9.0)$ & - & $10(3.9)$ \\
\hline Cat 4 & $3(7.1)$ & - & $3(3.0)$ & $3(3.3)$ & $9(3.5)$ \\
\hline
\end{tabular}

Values are presented as number $(\%)$. BS, Busan regional cancer center; CB, Chungbuk regional cancer center; IC, Incheon regional cancer center; NCC, National cancer center; Cat 1, Lung-RADS category 1; Cat 2, Lung-RADS category 2; Cat 3, Lung-RADS category 3; Cat 4, Lung-RADS category 4.

rent smokers group. However, the difference was not statistically significant. Also current smokers were less educated and had lower income than former smokers. However, the differences were not statistically significant.

\section{LDCT results}

Of the 256 participants in the pilot test, 19 (7.4\%) had positive screening results; $10(3.9 \%)$ were category 3 and nine $(3.5 \%)$ were category 4 . One participant $(0.4 \%)$ was diagnosed as lung cancer. The false-positive rate was $94.7 \%$. Table 2 demonstrates LDCT results by participating screening hospitals in pilot test. No adverse event after a diagnostic procedure is reported.

\section{Psychological harm}

Participant's anxiety of lung cancer had been slightly increased (Fig. 4). The mean score of anxiety was 4.2 (95\% confidence interval, 3.8 to 4.6$)$ before screening but increased to 4.7 (95\% confidence interval, 4.3 to 5.1) after screening and the difference was statistically significant $(\mathrm{p}=0.031)$.

\section{Willingness to quit smoking after screening}

Of the 256 participants, 138 were current smokers. Among them 28 did not answer in survey. Results shows an analysis of remaining 110 currently smoking participants.

Lung cancer screening participation and smoking cessation counselling had a positive effect on increasing participant's willingness to quit smoking on average (Table 3). The participant's willingness to quit smoking increased by $12.7 \%$ after lung cancer screening and the change was statistically significant $(\mathrm{p}=0.007)$. Although not statistically significant, participants with higher income level showed more willingness to quit smoking after screening than lower income

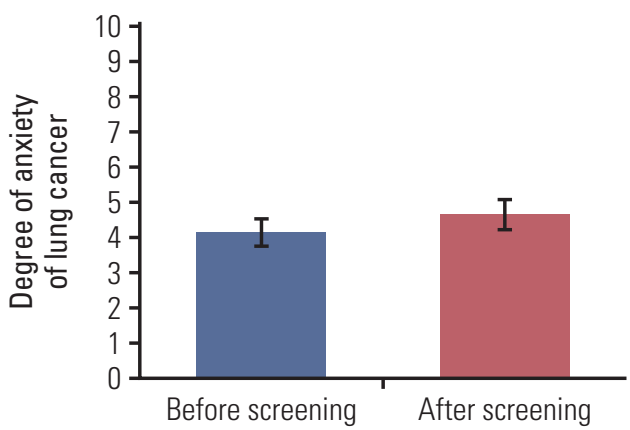

Fig. 4. Anxiety of lung cancer before and after lung cancer screening.

group. Changes in willingness to quit smoking also seemed greater for participants with less smoking history and more education group.

\section{Participant satisfaction with screening process}

Participants said they were generally satisfied with lung cancer screening process. Of 256 total participants, only 21 $(8.2 \%)$ said they were unsatisfied with general screening procedure. Among participants, $221(86.3 \%)$ said they are willing to participate in lung cancer screening again and $197(77.0 \%)$ said they are willing to recommend lung cancer screening to other people.

\section{Discussion}

This study reports the population based lung cancer screening protocol developed by multidisciplinary expert 
Table 3. Changes in willingness to quit smoking before and after lung cancer screening by participant characteristics

\begin{tabular}{|c|c|c|c|c|c|}
\hline \multirow{2}{*}{ Characteristic } & \multirow{2}{*}{ No. } & \multicolumn{2}{|c|}{ Mean (95\% CI) } & \multirow{2}{*}{ Change $(\%)$} & \multirow{2}{*}{ p-value ${ }^{a}$} \\
\hline & & Before screening & After screening & & \\
\hline Total & 110 & $6.3(5.8-6.9)$ & $7.1(6.6-7.6)$ & 12.7 & 0.007 \\
\hline \multicolumn{6}{|l|}{ Age group (yr) } \\
\hline $55-64$ & 86 & $6.3(5.7-6.9)$ & $7.3(5.7-6.8)$ & 15.8 & 0.002 \\
\hline $65-74$ & 24 & $6.5(5.2-7.8)$ & $6.3(5.1-7.6)$ & -3.1 & 0.753 \\
\hline \multicolumn{6}{|c|}{ Smoking history (pack year) } \\
\hline$<40$ & 50 & $6.7(5.9-7.4)$ & $7.6(7.0-8.1)$ & 13.4 & 0.002 \\
\hline$\geq 40$ & 60 & $6.0(5.2-6.9)$ & $6.7(5.9-7.5)$ & 11.7 & 0.147 \\
\hline \multicolumn{6}{|c|}{ Years of education } \\
\hline$\leq 9$ & 31 & $6.7(5.6-7.7)$ & $7.3(6.4-8.1)$ & 9.0 & 0.149 \\
\hline $10-12$ & 39 & $6.3(5.4-6.1)$ & $7.2(6.1-8.2)$ & 14.3 & 0.161 \\
\hline$\geq 13$ & 38 & $6.1(5.1-7.1)$ & $7.0(6.2-7.8)$ & 14.8 & 0.054 \\
\hline \multicolumn{6}{|c|}{ Monthly household income (million won) } \\
\hline$\leq 199$ & 46 & $6.6(5.7-7.4)$ & $6.8(6.0-7.6)$ & 3.0 & 0.427 \\
\hline 200-399 & 32 & $5.6(4.6-6.7)$ & $6.6(5.9-7.3)$ & 17.9 & 0.025 \\
\hline$\geq 400$ & 28 & $6.6(5.4-7.8)$ & $8.0(6.8-9.2)$ & 21.2 & 0.125 \\
\hline
\end{tabular}

$\mathrm{CI}$, confidence interval. ${ }^{\text {a) }}$ The changes in willingness to quit smoking before and after low-dose computed tomography screening were evaluated by paired $t$ test.

committee and the results of a small pilot test which evaluated the feasibility and compliance of the protocol. The protocol will be adopted to K-LUCAS which will be conducted by nationwide, multicenter study for evaluating the effectiveness and feasibility to implement nationwide lung cancer screening program.

The lung cancer screening program only focuses on highrisk populations aged between 55-74 years old current and former smokers (who quit smoking within 15 years) with at least 30 pack-years of smoking history which is recommended by NCC's guideline of lung cancer screening using LDCT [11].

The participants in K-LUCAS are recruited from the visitors participating in National Cancer Screening Program or smoking cessation services who are mandatorily required to complete a series of questionnaires. The questionnaires consist of a series of questions for the purpose of gathering information on demographics, smoking status, smoking history and health status including cancer history which are difficult to assess otherwise and enable us to apply inclusion and exclusion criteria.

A diagnostic accuracy and quality control is the key concern if the National Cancer Screening Program was implemented, especially in Korea where tuberculosis incidence rate is higher than most Organisation for Economic Cooperation and Development (OECD) countries [17]. K-LUCAS evaluates the capability of distinguishing pulmonary nodules from tuberculosis granulomas or scares, and the false-positive rate from screening. Failure to do so would result in high false-positives which may cause unnecessary invasive diagnostic procedures. In an attempt to improve diagnostic accuracy and quality control K-LUCAS has standardised screening results by Lung-RADS in support of network-based diagnosis supporting system using CAD program.

A small pilot test was conducted in four regional cancer hospitals to evaluate the feasibility and compliance of the protocol developed for K-LUCAS. First, we considered the positive rates and false-positive rates. Only $7.4 \%$ of participants had positive screening results based on Lung-RADS (Lung-RADS category 3 or above) and the false-positive rates was $94.7 \%$. The positive rates and the false-positive rates after first screening was $27.3 \%$ and $96.2 \%$ in NLST, respectively [8]. Our network-based diagnostic procedure involving Lung-RADS seems applicable overwhelming the initial worries of high tuberculosis incidence rates in Korea.

Second, smoking counselling service seems effective. A recent evidence shows that lung cancer screening can be perceived as a substitute for smoking cessation [19]. A provision of smoking cessation counselling to currently smoking participants would prevent a possible negative implication of lung cancer screening and promote a long-term benefit of lung cancer prevention by encouraging smoking cessation [20]. In the pilot test, lung cancer screening with LDCT had a positive impact on participant's willingness to quit smoking. The participant's motivation to quit smoking increased 
by $12.7 \%$ after screening on average.

Despite some promising aspects from the findings of the pilot test, some still remains controversial. For example, the participation rates in pilot test was only $61.5 \%$. A strategy to improve participation rates should be considered in K-LUCAS as the volume of participation would be directly linked to effectiveness of a national screening program if implemented. Previous evidence indicates this would be challenging because smokers are less likely to participate in cancer screening than non-smokers due to their pessimistic and fatalistic beliefs of health [21-23].

The anxiety of having lung cancer after LDCT screening increased by $11.9 \%$ on average in the current pilot test despite all participants visited the screening hospital to receive a face-to-face LDCT results counselling. However, one should be cautious about drawing any impulsive conclusion from this result as more investigation on psychological harm of lung cancer screening should be undertaken. For example, it would be interesting to see whether anxiety of having lung cancer diminishes over time but there was no follow-up period in the pilot test. More investigation on harm of lung cancer screening with LDCT will be undertaken in K-LUCAS [24].

Lastly, only $8.2 \%$ of total participants in the pilot test said they were unsatisfied with screening process in general and $86.3 \%$ of total participants said they are willing to participate in lung cancer screening again in the future. The participant compliance to the lung cancer screening protocols appears good.

The developed protocol in this study for K-LUCAS is assessed to be feasible for us to undertake a population-based lung cancer screening trial with substantially larger sample size based on the evidence in the pilot test. More discussion on the feasibility and the effectiveness of implementing a national lung cancer screening program will be followed in K-LUCAS. For example, the feasibility of participant selection and the quality control will be prioritised in K-LUCAS which was limited here due to small number of participants.

\section{Conflicts of Interest}

Conflict of interest relevant to this article was not reported.

\section{Acknowledgments}

This study is funded by a grant (1732130-1) from the National R\&D program for cancer control, Republic of Korea.

\begin{abstract}
Author Details
${ }^{1}$ National Cancer Control Institute, National Cancer Center, Goyang, ${ }^{2}$ Department of Cancer Control and Population Health, Graduate School of Cancer Science and Policy, National Cancer Center, Goyang, ${ }^{3}$ Department of Diagnostic Radiology, National Cancer Center, Goyang, Departments of ${ }^{4}$ Radiology and ${ }^{5}$ Internal Medicine, Seoul National University College of Medicine, Seoul, 'Department of Pulmonary, Allergy and Critical Care Medicine, Hallym University Sacred Heart Hospital, Anyang, ' ${ }^{7}$ epartment of Preventive Medicine, College of Medicine, The Catholic University of Korea, Seoul, ${ }^{8}$ Department of Internal Medicine, Chungbuk National University College of Medicine, Cheongju, 'Department of Family Medicine, Gachon University Gil Medical Center, Incheon, ${ }^{10}$ Department of Internal Medicine, Pusan National University, Busan, Korea
\end{abstract}

\section{References}

1. International Agency for Research on Cancer. Fact Sheets by Cancer [Internet]. Lyon: International Agency for Research on Cancer; c2018 [cited 2018 Jun 18]. Available from: http:// globocan.iarc.fr/Pages/fact_sheets_cancer.aspx.

2. Jung KW, Won YJ, Kong HJ, Lee ES; Community of Population-Based Regional Cancer Registries. Cancer statistics in Korea: incidence, mortality, survival, and prevalence in 2015. Cancer Res Treat. 2018;50:303-16.

3. Swensen SJ, Jett JR, Sloan JA, Midthun DE, Hartman TE, Sykes $\mathrm{AM}$, et al. Screening for lung cancer with low-dose spiral computed tomography. Am J Respir Crit Care Med. 2002;165:50813.

4. Henschke CI, Miettinen OS, Yankelevitz DF, Libby DM, Smith JP. Radiographic screening for cancer. Proposed paradigm for requisite research. Clin Imaging. 1994;18:16-20.
5. Kaneko M, Eguchi K, Ohmatsu H, Kakinuma R, Naruke T, Suemasu K, et al. Peripheral lung cancer: screening and detection with low-dose spiral CT versus radiography. Radiology. 1996;201:798-802.

6. Henschke CI, McCauley DI, Yankelevitz DF, Naidich DP, McGuinness G, Miettinen OS, et al. Early Lung Cancer Action Project: overall design and findings from baseline screening. Lancet. 1999;354:99-105.

7. Fontana RS, Sanderson DR, Taylor WF, Woolner LB, Miller WE, Muhm JR, et al. Early lung cancer detection: results of the initial (prevalence) radiologic and cytologic screening in the Mayo Clinic study. Am Rev Respir Dis. 1984;130:561-5.

8. National Lung Screening Trial Research Team, Aberle DR, Adams AM, Berg CD, Black WC, Clapp JD, et al. Reduced lung-cancer mortality with low-dose computed tomographic 
screening. N Engl J Med. 2011;365:395-409.

9. U.S. Preventive Services Task Force. Final recommendation statement: lung cancer: screening [Internet]. Rockville, MD: US Preventive Services Task Force; c2018 [cited 2018 Jun 18]. Available from: https://www.uspreventiveservicestaskforce. org/Page/Document/RecommendationStatementFinal/lung -cancer-screening.

10. Centers for Medicare \& Medicaid Services. Decision Memo for Screening for Lung Cancer with Low Dose Computed Tomography (LDCT) (CAG-00439N) [Internet]. Baltimore, MD: Centers for Medicare \& Medicaid Services; c2018 [cited 2018 Jun 18]. Available from: https://www.cms.gov/medicare-coverage-database/ details/nca-decision memo.aspx?NCAId=274.

11. Jang SH, Sheen SS, Kim HY, Yim HW, Park BY, Kim JW, et al. The Korean guideline for lung cancer screening. J Korean Med Assoc. 2015;58:291-301.

12. Eberth JM. Lung cancer screening with low-dose CT in the United States. J Am Coll Radiol. 2015;12(12 Pt B):1395-402.

13. Lee JW, Kim HY, Goo JM, Kim EY, Lee SJ, Kim TJ, et al. Radiological report of pilot study for the Korean Lung Cancer Screening (K-LUCAS) Project: feasibility of implementing lung imaging reporting and data system. Korean J Radiol. 2018;19: 803-8.

14. American College of Radiology. Lung Imaging Reporting and Data System (Lung-RAD) [Internet]. Preston, VA: American College of Radiology; c2018 [cited 2018 Jun 18]. Available from: https://www.acr.org/Clinical-Resources/Reportingand-Data-Systems / Lung-Rads.

15. McKee BJ, Regis SM, McKee AB, Flacke S, Wald C. Performance of ACR Lung-RADS in a clinical CT lung screening pro- gram. J Am Coll Radiol. 2015;12:273-6.

16. Huang P, Park S, Yan R, Lee J, Chu LC, Lin CT, et al. Added value of computer-aided CT image features for early lung cancer diagnosis with small pulmonary nodules: a matched casecontrol study. Radiology. 2018;286:286-95.

17. World Health Organization. Global tuberculosis report 2017. Geneva: World Health Organization; 2017.

18. Bach PB, Mirkin JN, Oliver TK, Azzoli CG, Berry DA, Brawley $\mathrm{OW}$, et al. Benefits and harms of CT screening for lung cancer: a systematic review. JAMA. 2012;307:2418-29.

19. Taylor KL, Cox LS, Zincke N, Mehta L, McGuire C, Gelmann E. Lung cancer screening as a teachable moment for smoking cessation. Lung Cancer. 2007;56:125-34.

20. Ostroff JS, Buckshee N, Mancuso CA, Yankelevitz DF, Henschke CI. Smoking cessation following CT screening for early detection of lung cancer. Prev Med. 2001;33:613-21.

21. Byrne MM, Davila EP, Zhao W, Parker D, Hooper MW, CabanMartinez A, et al. Cancer screening behaviors among smokers and non-smokers. Cancer Epidemiol. 2010;34:611-7.

22. Carreras G, Iannucci L, Costa G, Chellini E, Gorini G. Are smokers less likely to seek preventive healthcare measures in Italy? Eur J Cancer Prev. 2018;27:507-13.

23. Quaife SL, McEwen A, Janes SM, Wardle J. Smoking is associated with pessimistic and avoidant beliefs about cancer: results from the International Cancer Benchmarking Partnership. Br J Cancer. 2015;112:1799-804.

24. Wu GX, Raz DJ, Brown L, Sun V. Psychological burden associated with lung cancer screening: a systematic review. Clin Lung Cancer. 2016;17:315-24. 